\title{
Design and Performance Analysis of Radio over Fiber System Incorporating Differential Phase Shift Keying Modulation in High Speed Transmission System
}

\author{
NimishKumarSrivastava ${ }^{1}$, Arvind Kumar Jaiswal $^{2}$, Mukesh Kumar $^{3}$ \\ Department of Electronics and Communication EngineeringSam Higginbottom Institute of Agriculture, \\ Technology and Science, Allahabad, Uttar Pradesh, India -211007 \\ $1_{\text {PG Student }}, 2_{\text {Professor }}, 3_{\text {Assistant Professor }}$
}

\begin{abstract}
The fast growth of mobile-cellular telephone subscribers and mobile or fixed broadband internet users has triggered a traffic explosion and raised the demands for high broadband capacity and wide coverage network. Radio over fiber is an integration of wireless and fiber optic networks that provides untethered access to broadband wireless communications in a range of applications including last mile solutions, extension of existing radio coverage and capacity, and back haul.In this paper, after investigating the variations in $Q$ factor, $B E R$ and eye opening with respect to the wavelength, bit rate and fiber length we have compared DPSK,MSK,CPFSK and OQPSK modulation techniques used in RoF system for a bit rate in Gbps.
\end{abstract}

Keywords:CPFSK(Continuous Phase Frequency Shift Keying), DPSK(Differential Phase Shift Keying), MSK(Minimum Shift Keying), OQPSK(Offset Quadrature Phase Shift Keying), ROF(Radio Over Fiber).

\section{Introduction}

Radio-over-fiber(RoF) technology entails the use of optical fiber links to distribute radio frequency (RF) signals from a central location to the remote antenna units (RAUS)[1] . RoF systems with high data rates are used for wireless broadband communications as they can utilize the low loss and ultra wide bandwidth provided by optical fiber.The main advantage of RoF is low attenuation , large bandwidth, immunity to radio frequency interference, reduced power consumption, multi-operator and multi-service operation, dynamic resource allocation etc. Hence, it is more preferably compared to RF signal processing. The explosion in demand for network bandwidth is largely due to the growth in traffic such as video on demand, internet usages, and voice over IP, steaming video and voice.

Radio Over Fiber is basically an analog optical link which transmits modulated RF signals. It transmits RF signal downlink and uplink, to and from central station(CS) and to base station (BS) .The main requirements of a ROF link architecture are duplex operation (downlink-uplink), reasonable length and high performance optical components[1].

A Basic RoF system is described in Figure 1. In the downlink transmission, RF signals modulate the laser diode directly and results in intensity modulated optical signals at the CS. After that, they are transmitted through an optical fiber to the BS. At the BS, the signals are demodulated directly employing a photodiode for recovering the RF signals. Furthermore, they are amplified and radiated by an antenna. Based on modulation and detection perspective , RoF technology is known as intensity modulation and direct-detection (IMDD). The opposite process is carried out in the uplink transmission i.e. at the BS , the RF signals from the antenna directly modulate the laser diode and then the resulting optical signals are transmitted through an optical fiber to the CS. At the CS, the intensity modulated optical signals are demodulated directly employing a PD for recovering the RF signals. After that, the signals are amplified and further processed . 


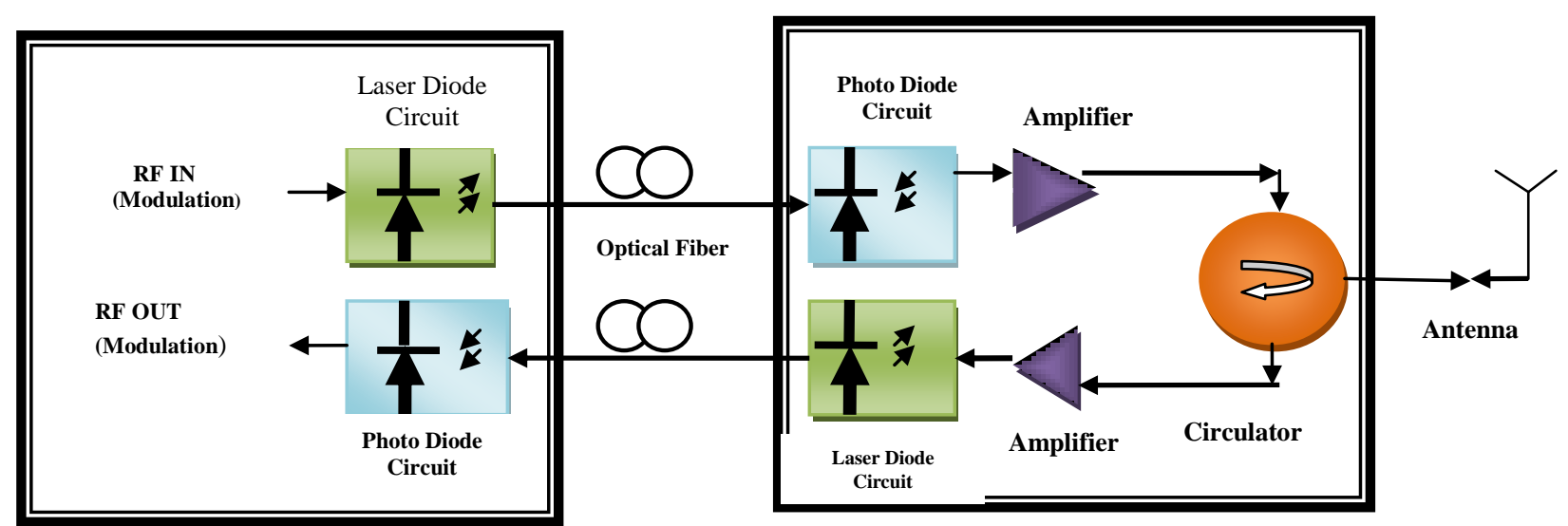

CENTRAL STATION

BASE STATION

Figure 1 : Basic RoF system

\section{Rof Design Using Optisystem}

Fig.3(a),3(b),3(c),3(d) shows the experimental set up of RoF system using DPSK,OQPSK,MSK and CPFSK modulation techniques. The pseudorandom bit sequence (PRBS) generator generate baseband $\operatorname{signal}\left(\mathrm{f}_{\mathrm{B}}\right)$ which is used to modulate a high frequency RF carrier of frequency $\mathrm{f}_{c}$ using electrical modulation which will shift this spectrum of data signal at frequencyf $f_{c}$. Differential Phase Shift Keying (DPSK) used for this electrical modulation is a non-coherent version of PSK ; it eliminates the need for a coherent reference signal at the receiver. Electrically modulated signal is then passed through band pass filter to remove unwanted frequency components. This filtered signal is used to modulate carrier light of a laser diode basedoptical source having frequency $f_{o}$ using an external modulator called machzehnder modulator (MZM). This optical modulation will again shift vertical axis of electrically modulated signal at frequency $f_{o}$ and the frequency of upper sideband of the optical signal will become fo+ fc. Now lower and upper frequencies of this upper sideband will become fo $+f_{c}+f_{B}$ and $f_{o}+f_{c}-f_{B}$ [2]. This optical upper sideband can be filtered using an optical band pass filter(OBPS) having frequency $\mathrm{f}_{\mathrm{o}}+\mathrm{f}_{\mathrm{C}}$ and bandwidth $1.5 * \mathrm{f}_{\mathrm{B}}$ (Bit rate). Subsequently ,this optical signal is transmitted through the channel based on single mode fiber. At the receiving end an optical amplifier is passed through optical band pass filter (OBPF) to filter the upper sideband of optical signal which is then applied to the PIN photo-detector. This photodetector demodulate filtered optical signal and convert this optical signal directly into a baseband signal. A low pass filter is used to remove a high frequency components and at the output of LPF data signal is detected and output is observed using BER Analyzer and Eye Diagram Analyzer[3],[4].

\section{III.Experimental Setup}

Fig. 3(a),3(b),3(c),3(d) shows the simulation setup for two users. Two pseudo random bit sequence(PRBS) generators are used to modulate two different data signals. These data are used to modulate two different electrical carriers having frequencies $250 \mathrm{GHz}$ and $255 \mathrm{GHz}$. These signals after passing through OBPF are combined using electrical power combiner and this combined signal is used to modulate an optical carrier of frequency 193.1THZ using mach-zehnder modulator(MZM). This modulated signal after passing through SMF is amplified using optical amplifier and then an optical power splitter[5] is used to split this optical signal into two signals. These optical signals are then passed through optical band pass filter ( frequencies 193.119THz, $193.120 \mathrm{THz}$ respectively and bandwidth $=1.5 *$ Bit rate). These filtered signals are then passed through photo-detector which will convert these optical signals directly into baseband signals. Low pass filters(Cutoff frequency $=.75 *$ Bit rate $\mathrm{Hz}$ ) are used to filter higher frequency components and finally we will get data which was initially transmitted[6],[7],[8]. 


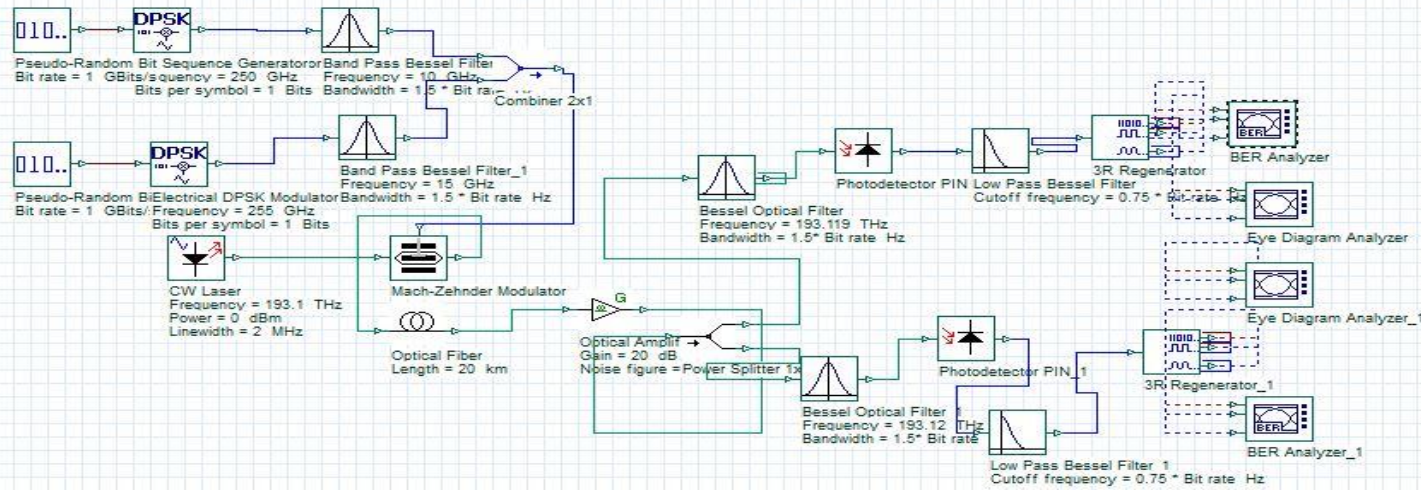

Figure 3(a)RoF system using DPSK technique

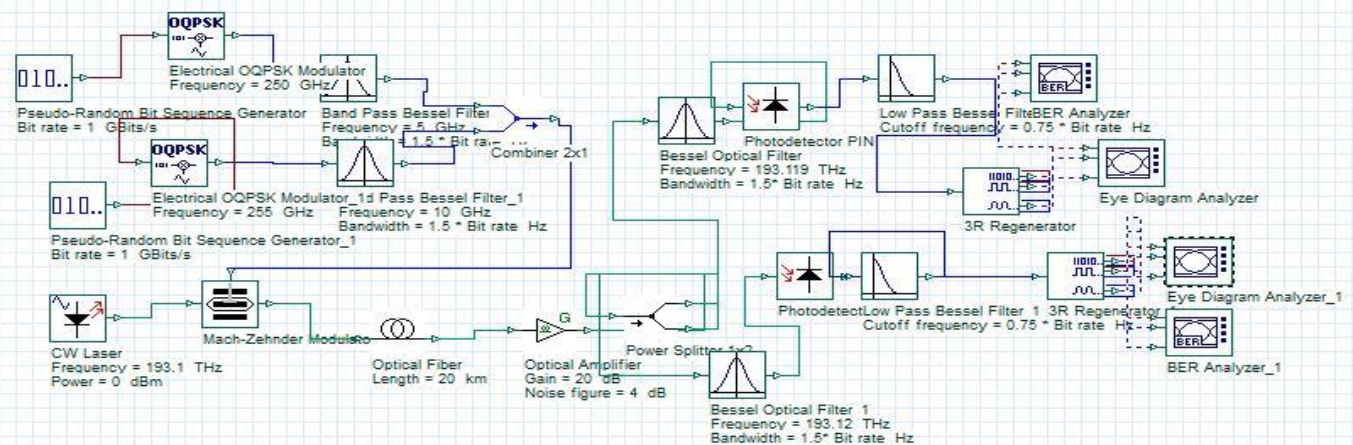

Figure 3(b)RoF system using OQPSK technique

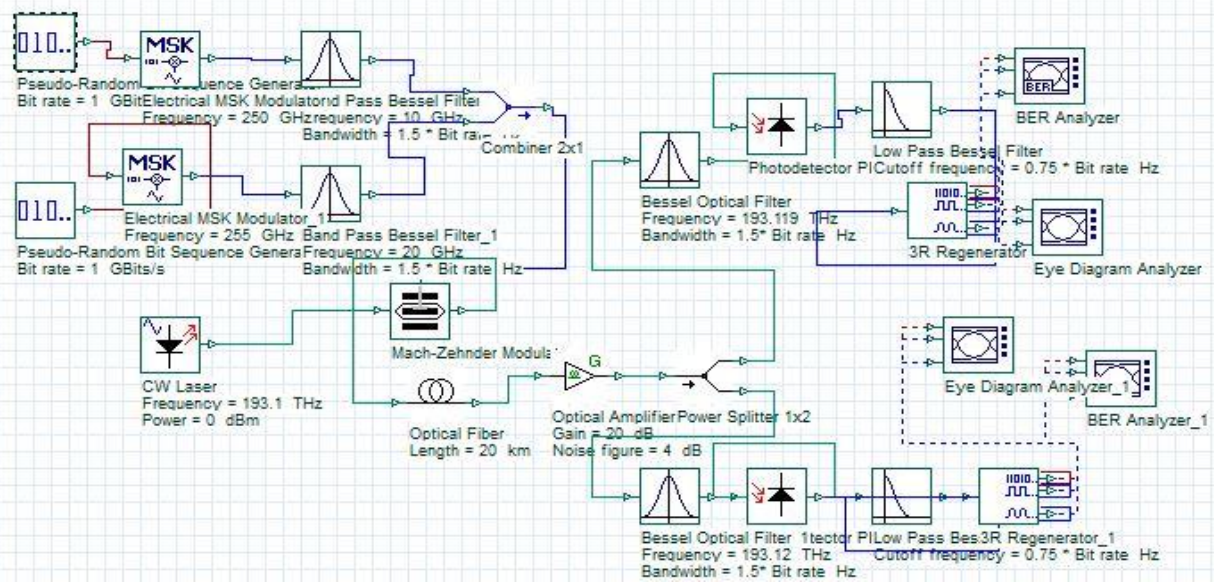

Figure3(c)RoF system using MSK technique

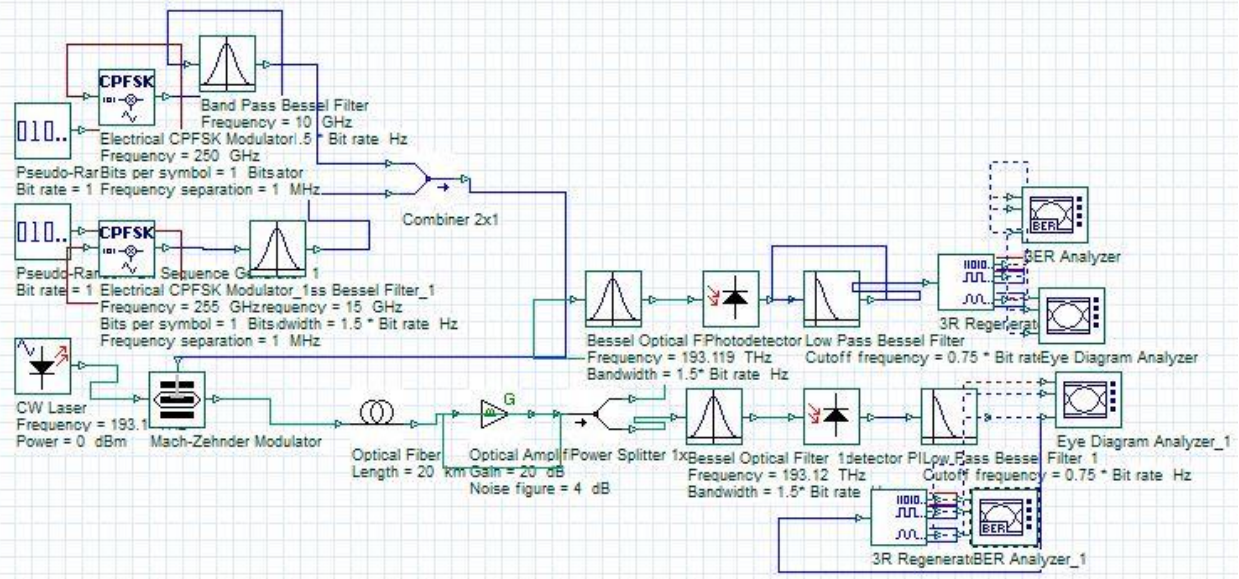

Figure3(d)RoF system using CPFSK technique 


\section{Result}

Fig.4(a), 4(b),4(c), 4(d) indicate the Quality factor and Bit Error Rate analysis of the system using eye diagram under Differential Phase Shift Keying, Offset Quadrature Phase Shift Keying, Minimum Shift Keying and Continuous Phase Frequency Shift Keying respectively. The observation are summarized in Table 4(1). As the Quality factor is a measure of the selectivity of circuit ; higher the circuit Q, smaller the bandwidth and thereby increasing the possibility of number of users in the system. Comparative studies performed indicates that DPSK modulation technique is best for Radio Over Fiber system because of highest quality factor and minimum bit error rate.

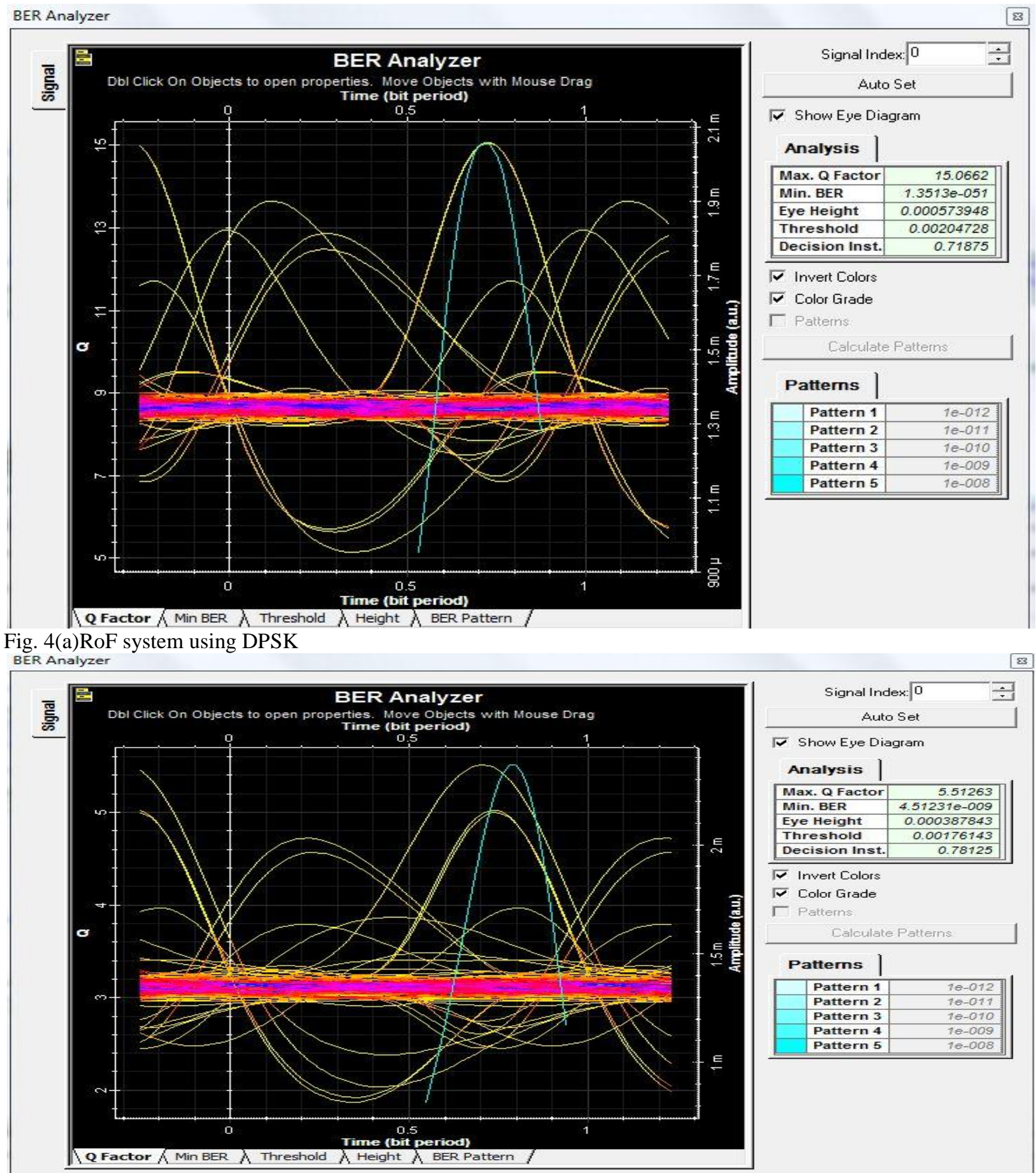

Fig. 4(b)RoF system using OQPSK technique 


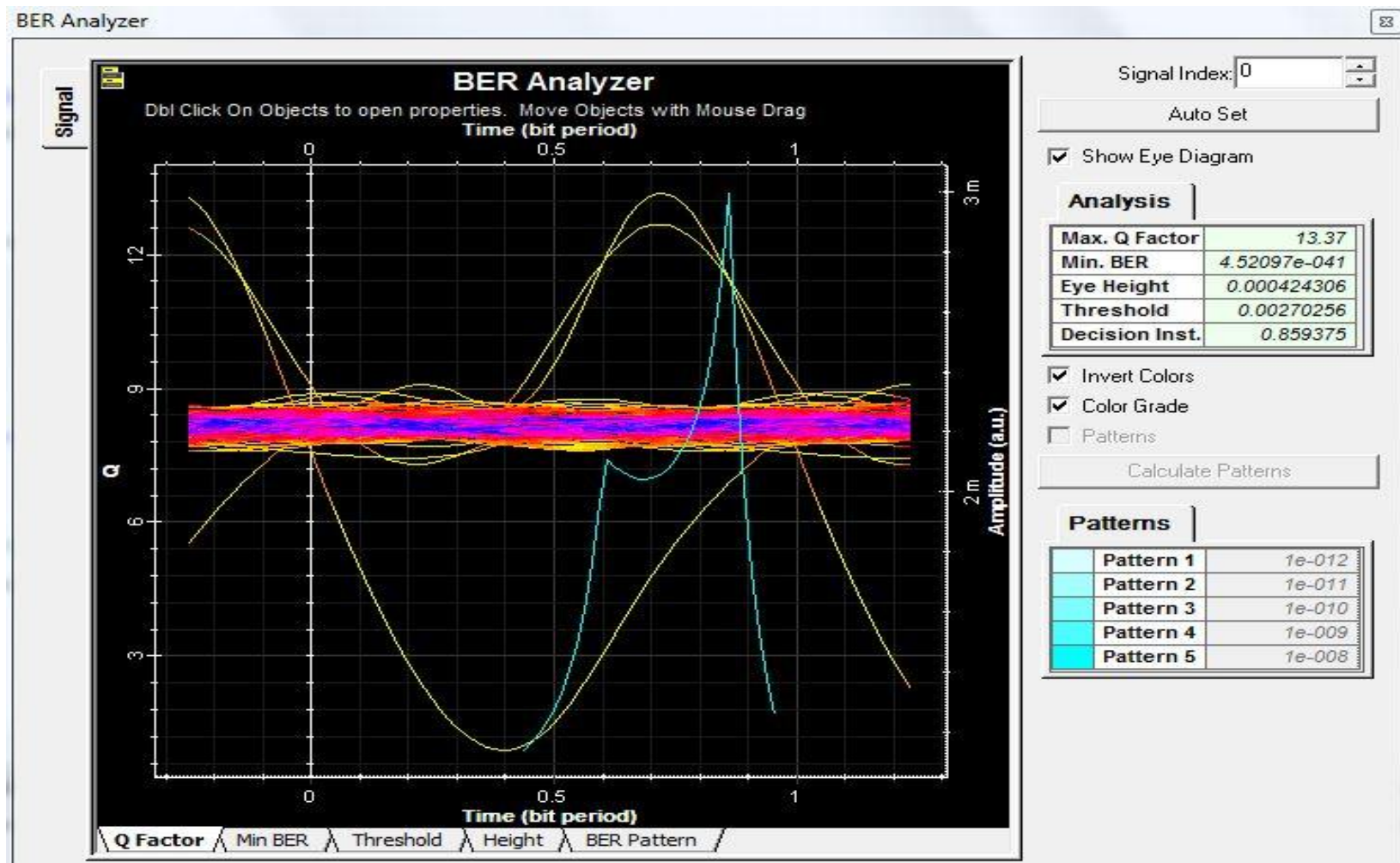

Fig. 4(c)RoF system using MSK technique

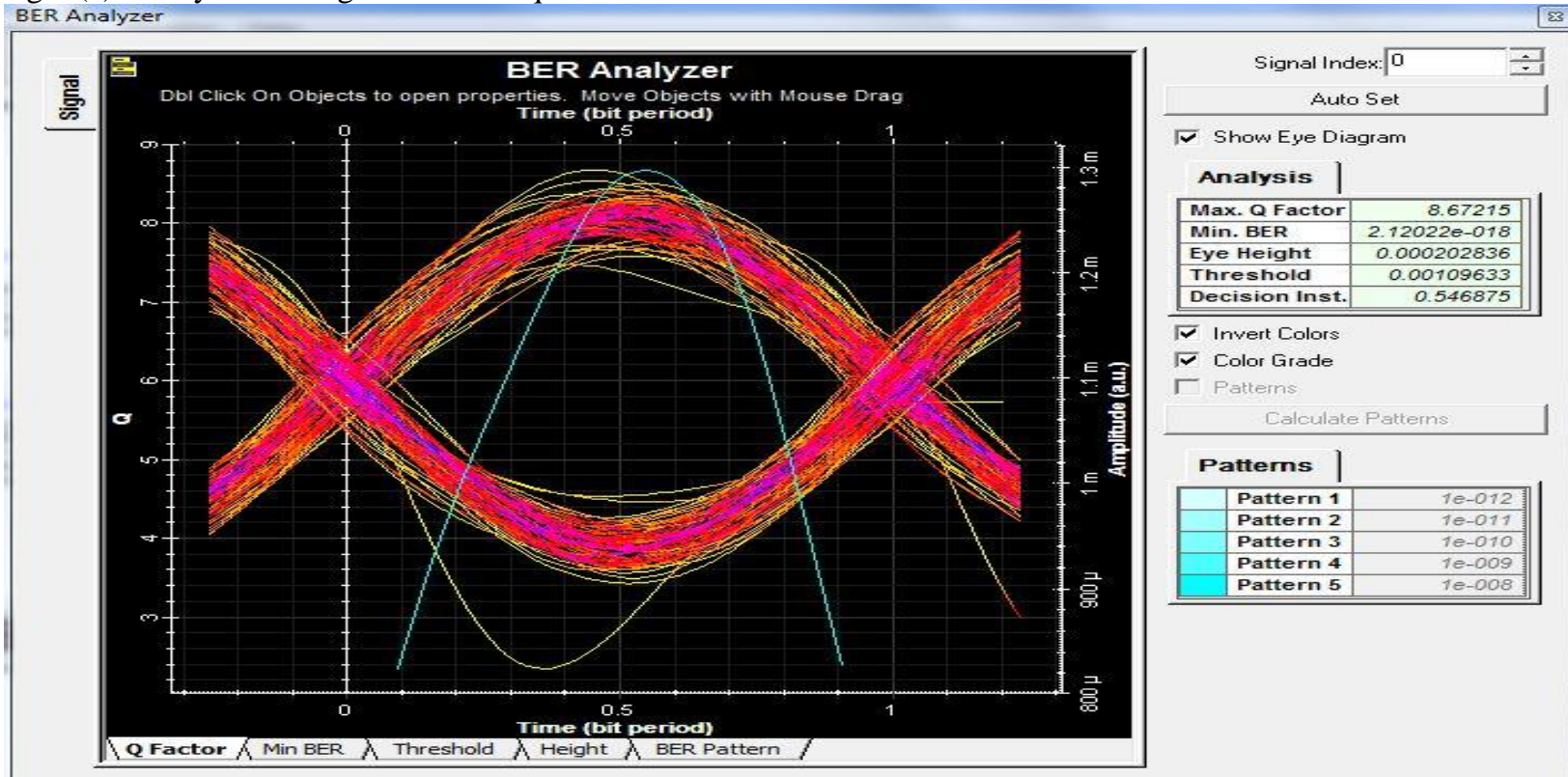

Fig. 4(d)RoF system using CPFSK technique

4(1) Table showing performance of different modulation technique

\begin{tabular}{|c|c|c|c|c|}
\hline \multirow{2}{*}{ Parameter } & \multicolumn{4}{|c|}{ Modulation Technique } \\
\hline & DPSK & OQPSK & MSK & CPFSK \\
\hline Maximum Q-Factor & 15.06 & 5.51 & 13.37 & 8.67 \\
\hline Minimum BER & $1.3 * 10^{-51}$ & $4.5 * 10^{-9}$ & $4.5 * 10^{-41}$ & $2.12 * 10^{-18}$ \\
\hline
\end{tabular}

\section{Conclusion}

A Radio over Fiber system has been designed and simulated using theoptisystem software and its parameters such as Q-factor and BER has been compared for different modulation techniques such as DPSK, OQPSK , MSK and CPFSK.RoF system with DPSK modulation and direct detection of baseband signal not only results in high performance but also brings in reduction in electronic components and elimination of 
electrical demodulation.Optical signal is directly converted into baseband signal using only one optical demodulation module at receiver. This makes the system simple, cheaper and more broadband. It is concluded that theRoF system incorporating DPSK modulation and direct detection of baseband signal has the potential to be applied in next generation convergent wireless-wired optical network.

\section{References}

[1] H.A. Mohammad, Performance evaluation of DWDM for Radio over Fiber system with Dispersion Compensation and EDFA , International Journal of Computer Applications, Vol.72, No.10, June 2013.

[2] J.Johny, S.Shashidharan, Design snd Simulation of a Radio Over Fiber System and its Performance Analysis, Optical Networking Technologies and Data Security- OPNTDS, 2012

[3] A.M. Zin , S.M. Idrus, The characterization of Radio over Fiber Employed GPON Architecture for Wireless Distribution Network , International Journal of Machine Learning and Computing, Vol.1, No.5, December 2011.

[4] S.M.J Alam, M.R. Alam, Bit Error Rate Optimization in Fiber Optic Communiations, International Journal of Machine Learning and Computing, Vol.1, No.5, December 2011.

[5] C.K. Wing, Y.Min, Radio-Over-Fiber 16-QAM , $100 \mathrm{~km}$ Transmission at 5Gb/s Using DSB-SC Transmitter and Remote Heterodyne Detection, Journal of Lightwave technology,Vol.26, No.6, March 15 ,2008.

[6] A.M.J. Koonen, M.G. Larrode, Perspectives of Radio over Fiber Technologies, OFC/NFOEC, 2008

[7] S.C.Chan, S.K. Hwang, J.M. Lin, Radio-over-Fiber AM-to-FM upconversion using an optically injected semiconductor laser, Optics Letters, Vol.31, No.15, August1 , 2006.

[8] H.B. Kim, M.Emmelmann, A Radio over Fiber Network Architecture for Road Vehicle Communication Systems, Proc. 47 ${ }^{\text {th }}$ IEEE Vehicular Technology Conference, 2005. 